\title{
Synthesis of hydrophobic resorcinol-formaldehyde
}

\section{xerogels by grafting with silanes}

Isabel D. Alonso-Buenaposada, M.A. Montes-Morán, J. Angel Menéndez, Ana

Arenillas*

Instituto Nacional del Carbón, CSIC, Apartado 73, 33080 Oviedo, Spain

\begin{abstract}
Hydrophilic organic xerogels were transformed into hydrophobic materials by treating them with hexamethyldisilazane. This transformation was performed by a simple one-pot process without modifying the previously designed porosity of the xerogel, at very soft operating conditions $\left(80^{\circ} \mathrm{C}\right.$ and atmospheric pressure) in the absence of catalyst or any other additional compound. Hexamethyldisilazane reacts with dangling hydroxyl surface groups, blocks the oxygen with methylsilane groups, and prevents the formation of hydrogen bonds with water molecules. The reaction proposed was corroborated chemically by X-ray photoelectron spectroscopy and thermogravimetric analysis coupled with a mass spectrometer. The porous structure of the organic xerogel determines the minimum time for the surface modification due to steric hindrance. Therefore, the former hydrophilic surface of the organic xerogel may be transformed into a hydrophobic one by a simple process, thus leading to the possibility of designing not only the porous structure but also the surface chemistry of the resorcinolformaldehyde xerogels to fit the requirements of an application.
\end{abstract}

Keywords: RF xerogels; hydrophobic materials; surface chemistry; porosity; silanes 


\section{Introduction}

Resorcinol-formaldehyde (RF) organic xerogels with a predetermined and purposedesigned pore texture can be obtained by selecting suitable physical and chemical variables during the synthesis process [1-3]. The use of microwave radiation as a heating source as an alternative to conventional synthesis offers the possibility of obtaining a tailored porous structure by a simple, fast, and cost-effective method suitable for producing RF xerogels on a large scale and has led to an increase in interest in these polymers [4].

Although the physical conditions used during the synthesis process may affect the porosity of the RF xerogels [5, 6], the size and volume of their pores are mainly determined by the following chemical variables: (i) the $\mathrm{pH}$ of the precursor solution [7]; (ii) the dilution ratio [8], i.e., the reactant/solvent ratio; (ii) the R/F molar ratio [9]; and (iv) the percentage of methanol used as stabilizer in the formaldehyde solution [10]. RF xerogels are usually thermally treated in an inert or reactive atmosphere to produce carbon materials called carbon xerogels or activated carbon xerogels [11, 12], which are useful for many applications because of their thermal and chemical stability. Under adequate synthesis conditions, these carbon materials preserve the porous structure of the RF precursors. The combination of these variables offers a wide range of possible porosities, thereby enabling the RF and carbon xerogels to be used in several very different applications (e.g., hydrogen storage, thermal insulation, as electrodes in supercapacitors, and as catalytic supports) [13-16].

RF xerogels not only have the advantage of a purpose-designed porosity but also of a very rich surface chemistry (i.e., a large oxygen surface group content) [17], making 
them suitable for a wide range of applications [18, 19]. Moreover, high-temperature posttreatments are not required for RF xerogels, thus leading to a notable reduction in the manufacturing costs. Furthermore, the high concentration of hydroxyl groups, which covers the surface of RF xerogels, provides them with a high hydrophilicity, which attracts moisture from the air, while their large pores improve their water sorption capacity, making them suitable for use as desiccant materials [17]. RF xerogels could also be used as thermal insulators because by tailoring their pore size and volume, it is possible to minimize their convective and conductive heat transfer capacities [20]. In this case, a hydrophilic surface in RF xerogels would be a disadvantage because the adsorbed water increases the conductivity of the material considerably; therefore, it would be mandatory to modify the original surface chemistry of RF xerogels to be used in this particular application. A hydrophobic surface is also clearly preferable when RF xerogels are used in applications such as adsorption or absorption of organic liquids and oils, transportation of nonpolar liquids in the nanoscale range in chemical and biotechnological applications, and adsorption of some toxic substances [21-22]. In these cases, the reversibility of the process itself would be even promoted by a reversibility of the surface chemistry of the materials.

In short, the possibility of tailoring the porosity (i.e., pore size and volume) of RF xerogels for a specific application is the main advantage of these synthetic materials. Nevertheless, the surface chemistry of RF xerogels may also play an important role, and the possibility of controlling their hydrophilic or hydrophobic nature will also be a determinant of their optimal performance in a specific application.

Several reports on a wide range of procedures for modifying hydrophilic surfaces into hydrophobic ones are already available in the literature. Some authors [23, 24] 
performed such surface modifications by using colloidal silica particles and fluoroalkylsilane as a silylating precursor in sol-gel processes. Another option reported in a previous study [25] is the preparation of a two-tier roughness surface using silicon, followed by the fluorination of the silicon surface. Other authors used dopamine to perform this in imitation of the technique of mussel coating [26], while few others used the chemical vapor deposition techniques [27] or plasma etching treatments [28]. Such hydrophobic polymeric surfaces can be produced by several techniques (i.e., layer-by-layer deposition, electrodeposition, electropolymerization, plasma and laser treatments, electrospining, etc. [29]), most of which are quite complex and tedious processes. Therefore, although it is possible to modify the surface nature of materials, this modification usually results in a considerable decrease in the cost-effectiveness of the materials.

Silica gels have hydroxyl groups covering their surface, which makes them a suitable and very commonly used desiccant material. The surface modification of silica gels has also been studied. The most common way of obtaining hydrophobic silica gels is to introduce methylsilane groups into the wet gel covering the surface, although other procedures such as postsynthesis treatments in liquid or gas phase can also be used $[30,31]$. The aims of such surface modifications are to prevent the collapse of pores during the drying step [32, 33], improve the diffusion of certain solvents [34, 35], and increase the durability of the material [36], among others. However, it is worth to be noted that these modifications usually modify the structure of the silica gel along with their surface chemistry. The compounds most commonly used to modify the surface of silica gels so that they become hydrophobic materials are trimethylchlorosilane (TMCS) [26, 37], trimethyl-methoxylated (TMMS) [28, 38], and 
hexamethyldisilazane (HMDZ) [32, 39]. Moreover, these silanes are also commonly used for the protection of alcohols in organic chemistry synthesis [40]. The ideal protecting group for an active hydrogen moiety such as alcohol would be one that could be introduced in a high yield and remain stable in certain conditions but could be selectively removed in a high yield in other conditions without modifying other functional groups present in the organic molecule. However, this kind of treatment with silanes always needs special operating conditions and the use of catalyst to promote the protection reaction.

The aim of the present work is to modify the hydrophilic surface of RF xerogels without (i) using complex processes that may affect the cost-effectiveness of the RF xerogels, (ii) without changing the controlled structure of the designed RF xerogels, and (iii) without using any other additive or catalysts that may affect the purity of the RF xerogels. These aims, to the best of our knowledge, have not been achieved before. To this end, RF xerogels were treated with silanes. To evaluate the possible effect of the pore size of the xerogel on the grafting process, two RF xerogels with very different porosities were evaluated and compared depending on the effectiveness (hydrophobic strength), time of treatment required, and treatment durability over time.

\section{Experimental Section}

\subsection{Synthesis of RF xerogels}

Two RF xerogels with average pore sizes of ca. 60 and $6000 \mathrm{~nm}$ (i.e., OX-60 and OX6000 , respectively) were evaluated for grafting with silanes. 
To obtain xerogel OX-60, $16.33 \mathrm{~g}$ of resorcinol (Indspec, 99\%) was dissolved in 83.36 $\mathrm{mL}$ of deionized water until complete dissolution under magnetic stirring. Subsequently, $100.31 \mathrm{~mL}$ of formaldehyde (37 wt.\% in water, stabilized by the addition of 12.5 wt.\% of methanol, supplied by Merck) was added. The $\mathrm{pH}$ of this solution was 3.25, and the proportions selected corresponded to a dilution ratio of 5.7 (i.e., the molar ratio of the total solvent to the reactants) and an $\mathrm{R} / \mathrm{F}$ molar ratio of 0.12 . In the case of OX-6000, $43.71 \mathrm{~g}$ of resorcinol (Indspec, 99\%) was dissolved in $110.38 \mathrm{~mL}$ of deionized water until complete dissolution under magnetic stirring. Subsequently, 45.90 $\mathrm{mL}$ of formaldehyde (37 wt.\% in water, stabilized by adding 0.7 wt.\% of methanol, supplied by Química S.A.U) was added. To this precursor solution, drops of a 5-M $\mathrm{NaOH}$ solution (prepared from AnalaR Normapur, 99.9\%) were added until a pH of 5 was obtained. The proportions selected corresponded to a dilution ratio of 8 and $\mathrm{R} / \mathrm{F}$ molar ratio of 0.7 .

Each precursor solution was placed in a microwave oven (in-lab designed and constructed [4]) at $85{ }^{\circ} \mathrm{C}$ for 3 hours to allow gelation and curing to occur. After the formation of the polymeric structure, all excess water was eliminated by heating the gel in the same device continuously until a mass loss of $50 \mathrm{wt} . \%$ was attained. This drying step lasted ca. 2 hours. Each material was kept in the oven at $100{ }^{\circ} \mathrm{C}$ overnight to remove any traces of moisture and unreacted compounds to obtain a completely stable material.

The final material was ground and sieved to $<212 \mu \mathrm{m}$ to obtain a homogeneous powder suitable for the treatments. 


\subsection{Grafting with silanes}

The following silanes were used for the RF xerogel grafting process: TMCS (Merck, >99\%), TMMS (Merck, >98\%), and HMDZ VLSI selectipur (BASF). All the silanes were used in the treatments as received. Suspensions of $8 \mathrm{~g}$ of each RF xerogel in 100 $\mathrm{mL}$ of each silane compound were stirred at $80^{\circ} \mathrm{C}$ and atmospheric pressure. Aliquots of the resulting material were extracted at different reaction times to measure the progression of the reaction. To remove any residual or nonreacted compounds from the porous structure of the samples, all the samples were degasified at $120{ }^{\circ} \mathrm{C}$ and vacuum overnight before any characterization. Furthermore, the samples were washed with hexane prior to the degasification procedure, to improve the elimination of physisorbed compounds; however, no differences were observed between washed and unwashed samples.

\subsection{Sample characterization}

\subsubsection{Porous properties characterization}

Before characterization, the samples were outgassed (Micromeritics VacPrep 0.61) at 0.1 mbar and $120{ }^{\circ} \mathrm{C}$ overnight to remove any humidity and other physisorbed gases. The textural properties were characterized by nitrogen adsorption-desorption isotherms, mercury porosimetry, and density analysis.

The nitrogen adsorption-desorption isotherms were measured at $-196{ }^{\circ} \mathrm{C}$ using a Tristar 3020 (Micromeritics) device. The pore volume and mean pore size were determined by mercury porosimetry, using an AutoPore IV 9500 (Micromeritics) from atmospheric pressure up to $228 \mathrm{MPa}$. The range of pore sizes was from 5.5 to $2000 \mathrm{~nm}$. The surface tension and contact angle were $485 \mathrm{mN} \mathrm{m}^{-1}$ and $130^{\circ}$, respectively, and the stem volume 
was between $45 \%$ and $60 \%$ in all the analyses. In the low-pressure step, the samples were evacuated up to $6.7 \mathrm{~Pa}$, and the equilibration time used was $10 \mathrm{~s}$. To determine the bulk density and the percentage of porosity of the samples, a Geopyc 1360 (Micromeritics) device was set to a Dryflow of $0.8 \mathrm{~g}$ and a sample volume of around $50 \%$ of the total volume. A chamber with an internal diameter of $12.7 \mathrm{~mm}$ and a strength of $28 \mathrm{~N}$ for 20 cycles with a conversion factor of $0.1284\left(\mathrm{~cm}^{3} \mathrm{~mm}^{-1}\right)$ was used.

\subsubsection{Chemical characterization}

The surface chemical modification was verified by two different analyses. On the one hand, X-ray photoelectron spectroscopy (XPS) of the RF xerogels was performed in a SPECS Phoibos 100 analyzer using MgK $\alpha$ X-rays $(1254.6 \mathrm{eV})$ at a power of $100 \mathrm{~W}$ and in a residual vacuum of $10^{-7} \mathrm{~Pa}$. Analyzer pass energy of $50 \mathrm{eV}$ was used to collect broad scan spectra $(0-1100 \mathrm{eV})$. The atomic percentages (atom\%) of the different elements present in an approximately 10-nm upper layer probed by XPS were calculated from the survey spectra by considering the integrated areas of the main XPS peaks. The high-resolution spectra of $\mathrm{Si}(2 \mathrm{p})$ were obtained using a pass energy of $10 \mathrm{eV}$.

On the other hand, temperature-programmed desorption (TPD) experiments were performed using a thermogravimeter (DSC Q600 TA Instruments) coupled with a mass spectrometer (ThermoStar Pfeiffer). The sample was heated in a thermobalance at $10^{\circ} \mathrm{C}$ $\min ^{-1}$ up to $700^{\circ} \mathrm{C}$, while the $\mathrm{m} / \mathrm{z}$ signals (from 1 to 100 ) were registered in the mass spectrometer.

\subsubsection{Contact angle determination}

To determine the hydrophobic nature of the RF xerogel surface, the contact angle between a drop of deionized water and the surface of the material was measured using a Krüss 62/G40 optical tensiometer. Each drop of water (ca. $6 \mathrm{~mm}$ in diameter) was 
deposited onto a completely flat surface using a micrometric syringe. A pellet of $10-\mathrm{mm}$ diameter was prepared from the sample powders $(<212-\mu \mathrm{m}$ particle size $)$ by applying a pressure of 10 Tons for $25 \mathrm{~s}$. In all the cases, three different pellets from each sample were evaluated, and the average of the contact angles was selected as the final value.

\section{Results and Discussion}

In this study, two RF xerogels with very different porosities were selected to be used in two different applications where hydrophobicity would be a required property: OX-60 with a pore size and properties suitable for use as a thermal insulator [18] and OX-6000 with a pore size and properties suitable for oil spill cleaning [41]. These two applications were selected because the porosity requirements for them are very different; however, the possible applications of the modified RF xerogels are enormous. Given that the surface chemistry of both samples selected was analogous [17], potential differences detected during the grafting process with silanes could be only because of the porosity of the samples. Table 1 shows the main porous characteristics of the two samples studied. As can be seen, both samples had a similar bulk density and open porosity (i.e., ca $0.3 \mathrm{~g} \mathrm{~cm}^{-1}$ and ca. $75 \%$, respectively) but very different pore sizes and volumes. Their microporosities also differed, with BET surface areas of 244 and $20 \mathrm{~m}^{2}$ $\mathrm{g}^{-1}$ for OX-60 and OX-6000, respectively.

The three silanes (TMCS, TMMS, and HMDZ) grafted onto the RF xerogels were tested under the same operational conditions. However, because of the acidic surface of the xerogels (i.e., $\mathrm{pH}_{\mathrm{PZC}}$ of around 3), reaction with TMCS and TMMS is not favored. A basic booster is required because an initial deprotonation reaction needs to occur before the grafting reaction starts. In contrast, the reaction with HMDZ was favored because of 
the basicity of this particular silane reagent $(\mathrm{pKa}=7.5)$. The nitrogen in HMDZ would extract the hydrogen from the hydroxyl groups in the RF xerogel, giving rise to their anionic form. This anionic group would then act as a nucleophile with the electron pair in the oxygen reacting with the silicon atoms in the $\mathrm{HMDZ}$ by a $\mathrm{SN}_{2}$ reaction, thus resulting in $\mathrm{O}-\mathrm{Si}$ bonds (see reaction mechanism in Figure 1). The ammonia produced in the reaction was eliminated as the reaction proceeded, and because of this, the resultant material contained no nitrogen groups. Consequently, a RF xerogel with a surface covered with nonpolar alkyl groups was obtained.

To corroborate the grafting reaction with HMDZ, chemical analyses were performed. RF xerogel OX-60 was selected as it was the sample with the narrowest pores, and therefore, a priori, the sample with greater steric hindrance for the reaction. Figure 2 shows the Si (2p) high-resolution spectra of the raw xerogel (OX-60) and the treated sample Si-OX-60. It is clear that the original sample did not show any presence of silica, as expected, while the treated sample presented a clear peak at $103.5 \mathrm{eV}$. Furthermore, the chemical composition obtained from XPS showed a 77.7 atomic $\%$ of carbon and 22.3 atomic $\%$ of oxygen for sample OX-60, while the treated sample, SiOX-60, presented a 78.6 atomic $\%$ of C, 19.4 atomic $\%$ of O, and 2 atomic $\%$ of Si.

Additionally, the temperature-programmed desorption performed in the thermobalance coupled with mass spectrometer corroborated the presence of silica functionalities in the surface of the treated sample Si-OX-60. From Figure 3, the evolution of the $\mathrm{m} / \mathrm{z} 75$, corresponding to the trimethylsyloxyl compounds $\left(\mathrm{C}_{2} \mathrm{H}_{7} \mathrm{SiO}^{+}\right)$, is clear for the grafted sample, and this $\mathrm{m} / \mathrm{z}$ signal was not detected for the raw material. Therefore, it is obvious that the grafting reaction had occurred, and the surface of the RF xerogel was 
modified. Moreover, this technique provides insight on the stability of the silanated functionalities passivating the surface. As shown in Figure 3, the decomposition of the silanated groups began at about $250{ }^{\circ} \mathrm{C}$, with its maximum release at $300{ }^{\circ} \mathrm{C}$. Therefore, it can be said that the passivation treatment is temperature resistant up to $250{ }^{\circ} \mathrm{C}$.

The effectiveness, of this chemical surface modification, on the surface affinity to water of the RF xerogels was evaluated by measuring the contact angle between a drop of deionized water and the surface of the material using an optical tensiometer. Figure 4 shows the contact angle measured at different reaction times for the different treatments. In the first stage, sample OX-6000 was treated with the three silanes, as this was the sample with, a priori, less steric hindrance for the surface modification. As can be seen, when using TMCS and TMMS as grafting agents, no change in the contact angle was detected for the resulting samples at any time of reaction. The contact angle for these samples, and for the pristine RF xerogel, was considered $0^{\circ}$ because the drops of water were quickly absorbed by the pellets. This indicates that both TMCS and TMMS did not react with the surface of the RF xerogels, and therefore, the materials remained hydrophilic after the treatment.

However, in the case of HMDZ, a change in the contact angle was observed (Figure 4; for brevity, the HMDZ-grafted OX-60 and OX-6000 are labelled Si-OX-60 and Si-OX6000, respectively). In fact, when a RF xerogel with large pore size (OX-6000) was treated with HMDZ, the hydrophilic $\left(\alpha<90^{\circ}\right)$ material changed to hydrophobic $(\alpha>$ $90^{\circ}$ ) after a grafting reaction of only 1 minute, and the maximum hydrophobicity (ca. $\alpha$ $\left.=125^{\circ}\right)$ was achieved after 8 minutes. In contrast, the OX-60 sample needed a longer reaction time, as can be seen from Figure 4, where no hydrophobicity was observed 
until after 1 hour of reaction. In this case, the maximum hydrophobicity, again $\alpha=125^{\circ}$, was attained only after $8 \mathrm{~h}$ of treatment. The difference in these grafting reaction times was because it was more difficult for HMDZ to diffuse along the narrow pores of OX60 than along the wider pores of OX-6000. However, the maximum hydrophobicity finally attained by both RF xerogels was the same because of the analogous surface chemistries of the samples.

The attachment of alkyl groups to the RF xerogel may cause changes in the porous properties of the samples. As mentioned before, the ability to tailor the porosity of the RF xerogels is the main advantage of these materials. Therefore, it is essential to check for possible changes in porosity after each treatment.

Figure 5 shows the pore size distributions of the original and grafted samples as measured by $\mathrm{Hg}$ porosimetry. An increase in the pore volume and mean pore size of the treated samples was observed after the treatment. The pore volume of Si-OX-6000 and Si-OX-60 was 2.08 and $0.97 \mathrm{~cm}^{3} \mathrm{~g}^{-1}$, respectively. This implies an increase in pore volume of 29\% for OX-6000 and 18\% for OX-60 (see Table 1). However, the average pore size increased from 59 to $70 \mathrm{~nm}$ in the case of Si-OX-60 and from 5694 to 6160 $\mathrm{nm}$ for Si-OX-6000. However, these increments in pore size and volume have to be treated with caution, as the RF xerogels studied have poor mechanical strengths, and therefore, they are very sensitive to the changes in pressure levels used in mercury porosimetry (i.e., the porosimeter could interpret pore volume when the sample is compressed). Other authors have observed that compression problems in the analyses of these materials can lead to overestimations of the pore volume and size [42], and these overestimations may be larger in the case of the modified materials because of the extra 
chains grafted. Thus, it should be concluded that the porosities of the RF xerogels in this study were not considerably affected by the grafting treatment applied. This can be seen from the fact that sample OX-60 and its counterpart have a pore volume of around 0.9 $\mathrm{cm}^{3} \mathrm{~g}^{-1}$ and a pore size of $60 \mathrm{~nm}$, while OX-6000 and its counterpart have twice the pore volume (ca. $2 \mathrm{~cm}^{3} \mathrm{~g}^{-1}$ ) and a pore size of around $6000 \mathrm{~nm}$.

Now that it has been shown that initially hydrophilic RF xerogels can be converted into hydrophobic materials without experiencing almost any change in their porous properties, it might be worthwhile to evaluate the modifications of the surface properties with time under atmospheric exposure. Therefore, both grafted samples were exposed to ambient conditions (i.e., $20{ }^{\circ} \mathrm{C}$ and $80 \%$ humidity), and the contact angle was checked periodically over a period of several days. Figure 6 confirms that the surface modification of both samples is not permanent. The hydrolysis of the silane surface groups due to the humidity in the atmosphere seems to be responsible for the gradual transition from hydrophobic back to hydrophilic xerogels. Trimethylsilanes are relatively easy to hydrolyze [40, 43]. Sample Si-OX-6000 became hydrophilic again after 19 days of exposure to ambient humid conditions. In the case of the sample with narrow pores, Si-OX-60, after 19 days, the sample remained hydrophobic $\left(\alpha>90^{\circ}\right)$, although a decrease in the contact angle was noticeable after 2 weeks. After 4 weeks of exposure of Si-OX-60 to ambient humid conditions, the surface was hydrophilic $(\alpha<$ $90^{\circ}$ ), presumably because of the loss of many alkyl surface groups introduced during the treatment with HMDZ, but it was still possible to measure a contact angle. However, after 35 days of ambient exposure, the material became highly hydrophilic again to the point that it resembled the initial sample (see Figure 6). In short, it can be said that the easier the alkyl groups are introduced, the easier they are removed, and the hydrolysis 
reaction of RF xerogels with HMDZ is favored, especially in the wider pore sample ( $\mathrm{Si}$ OX-6000).

Moreover, in both samples, Si-OX-60 and Si-OX-6000, the grafting degradation shown in Figure 6 seems to have occured in two stages. In the case of Si-OX-6000, the degradation of the external non-polar surface groups occured only after 2 days, and then, the sample remained stable, and hydrophobic, for nearly 4 more days before the hydrolysis of the grafted functionalities in the internal pore structure presumably occurred, after which the sample became hydrophilic. However, in the case of the sample Si-OX-60, a plateau was detected after the degradation of the most external grafted functionalities that occurred after 20 days; however, the sample cannot be considered hydrophobic at this stage because the contact angle detected was slightly lower than $90^{\circ}$.

Samples Si-OX-60 and Si-OX-6000 are hydrophobic at least for several days. These materials avoid any contact with water (Figure 7a). However, if the sample/water suspension is shaken overnight, a good dispersion of the sample can be obtained (Figure $7 b)$.

Thus, the surface modification of the RF xerogels was easily reversed by simply hydrolyzing the methylsilane groups. However, if kept away from moisture, their hydrophobicity remained intact. This property of RF xerogels could be very useful for applications, such as separation or transport of nonpolar compounds by adsorption, where the adsorbate can be easily desorbed by a simple process of water scrubbing.

\section{Conclusions}


The surface nature of RF xerogels can be easily converted from hydrophilic to hydrophobic by grafting HMDZ onto the RF xerogels in one simple step. No extra compounds or catalyst is needed. The duration of the modification process depends on the porous properties of the RF xerogel due to diffusional factors. The wider the pore size, the quicker is the process of obtaining a hydrophobic surface. The tailored porosity of the RF xerogels is not substantially modified by the treatments. Depending on the final application of the xerogels (i) their hydrophobicity can be maintained if exposure to moisture is avoided or (ii) it can be easily reversed by a simple hydrolysis process.

\section{Acknowledgments}

The authors gratefully acknowledge the financial support of the Ministerio de Economía y Competitividad of Spain, MINECO (Project CTQ2014-54772-P).

\section{References}

[1] S.A. Al-Muhtaseb, J.A. Ritter, Preparation and Properties of ResorcinolFormaldehyde Organic and Carbon Gels, Advanced Materials, 15 (2003) 101-114.

[2] F.L. Conceição, P.J.M. Carrott, M.M.L. Ribeiro Carrott, New carbon materials with high porosity in the 1-7 nm range obtained by chemical activation with phosphoric acid of resorcinol-formaldehyde aerogels, Carbon, 47 (2009) 1874-1877.

[3] S. Morales-Torres, F.J. Maldonado-Hódar, A.F. Pérez-Cadenas, F. Carrasco-Marín, Textural and mechanical characteristics of carbon aerogels synthesized by 
polymerization of resorcinol and formaldehyde using alkali carbonates as basification agents, Physical Chemistry Chemical Physics, 12 (2010) 10365-10372.

[4] E.G. Calvo, E.J. Juárez-Pérez, J.A. Menéndez, A. Arenillas, Fast microwave-assisted synthesis of tailored mesoporous carbon xerogels, Journal of Colloid and Interface Science, 357 (2011) 541-547.

[5] J. Menéndez, A. Arenillas, B. Fidalgo, Y. Fernández, L. Zubizarreta, E. Calvo, J. Bermúdez, Microwave heating processes involving carbon materials, Fuel Processing Technology, 91 (2010) 1-8.

[6] N. Rey-Raap, J.A. Menéndez, A. Arenillas, Optimization of the process variables in the microwave-induced synthesis of carbon xerogels, J Sol-Gel Sci Technol, 69 (2014) 488-497.

[7] N. Job, R. Pirard, J. Marien, J.-P. Pirard, Porous carbon xerogels with texture tailored by pH control during sol-gel process, Carbon, 42 (2004) 619-628.

[8] Z. Zapata-Benabithe, C. Moreno-Castilla, F. Carrasco-Marín, Effect of dilution ratio and drying method of resorcinol-formaldehyde carbon gels on their electrocapacitive properties in aqueous and non-aqueous electrolytes, Journal of Sol-Gel Science and Technology, 75 (2015) 407-412.

[9] N. Rey-Raap, J. Angel Menéndez, A. Arenillas, Simultaneous adjustment of the main chemical variables to fine-tune the porosity of carbon xerogels, Carbon, 78 (2014) 490-499.

[10] I.D. Alonso-Buenaposada, N. Rey-Raap, E.G. Calvo, J. Angel Menéndez, A. Arenillas, Effect of methanol content in commercial formaldehyde solutions on the porosity of RF carbon xerogels, Journal of Non-Crystalline Solids, 426 (2015) 13-18. 
[11] M.S. Contreras, C.A. Páez, L. Zubizarreta, A. Léonard, S. Blacher, C.G. OliveraFuentes, A. Arenillas, J.-P. Pirard, N. Job, A comparison of physical activation of carbon xerogels with carbon dioxide with chemical activation using hydroxides, Carbon, 48 (2010) 3157-3168.

[12] A.H. Moreno, A. Arenillas, E.G. Calvo, J.M. Bermúdez, J.A. Menéndez, Carbonisation of resorcinol-formaldehyde organic xerogels: Effect of temperature, particle size and heating rate on the porosity of carbon xerogels, Journal of Analytical and Applied Pyrolysis, 100 (2013) 111-116.

[13] L. Zubizarreta, J.A. Menéndez, N. Job, J.P. Marco-Lozar, J.P. Pirard, J.J. Pis, A. Linares-Solano, D. Cazorla-Amorós, A. Arenillas, Ni-doped carbon xerogels for H2 storage, Carbon, 48 (2010) 2722-2733.

[14] V. Celorrio, J. Flórez-Montaño, R. Moliner, E. Pastor, M.J. Lázaro, Fuel cell performance of Pt electrocatalysts supported on carbon nanocoils, International Journal of Hydrogen Energy, 39 (2014) 5371-5377.

[15] D. Fairén-Jiménez, F. Carrasco-Marín, C. Moreno-Castilla, Adsorption of Benzene, Toluene, and Xylenes on Monolithic Carbon Aerogels from Dry Air Flows, Langmuir, 23 (2007) 10095-10101.

[16] P. Staiti, A. Arenillas, F. Lufrano, J.Á. Menéndez, High energy ultracapacitor based on carbon xerogel electrodes and sodium sulfate electrolyte, Journal of Power Sources, $214(2012) 137-141$.

[17] I.D. Alonso-Buenaposada, E.G. Calvo, M.A. Montes-Morán, J. Narciso, J.A. Menéndez, A. Arenillas, Desiccant capability of organic xerogels: Surface chemistry vs porous texture, Microporous and Mesoporous Materials, 232 (2016) 70-76. 
[18] L. Pilato, Phenolic resins: 100 years and still going strong, Reactive \& Functional Polymers 73 (2013) 270-277.

[19] J.G. Handique, J.B. Baruah, Polyphenolic compounds: an overview, Reactive \& Functional Polymers 52 (2002) 163-188.

[20] E. Cuce, P.M. Cuce, C.J. Wood, S.B. Riffat, Toward aerogel based thermal superinsulation in buildings: A comprehensive review, Renewable and Sustainable Energy Reviews, 34 (2014) 273-299.

[21] T. Ning, G. Yang, W. Zhao, X. Liu, One-pot solvothermal synthesis of robust ambient-dried polyimide aerogels with morphology-enhanced superhydrophobicity for highly efficient continuous oil/water separation, Reactive and Functional Polymers, 116 (2017) 17-23.

[22] B. Kiskan, Adapting benzoxazine chemistry for unconventional applications, Reactive and Functional Polymers, doi.org/10.1016/j.reactfunctpolym.2017.06009 (2017).

[23] M. Hikita, K. Tanaka, T. Nakamura, T. Kajiyama, A. Takahara, Super-LiquidRepellent Surfaces Prepared by Colloidal Silica Nanoparticles Covered with Fluoroalkyl Groups, Langmuir, 21 (2005) 7299-7302.

[24] H.M. Shang, Y. Wang, S.J. Limmer, T.P. Chou, K. Takahashi, G.Z. Cao, Optically transparent superhydrophobic silica-based films, Thin Solid Films, 472 (2005) 37-43.

[25] X. Yao, L. Xu, L. Jiang, Fabrication and characterization of superhydrophobic surfaces with dynamic stability, Advanced Functional Materials, 20 (2010) 3343-3349.

[26] S.M. Kang, I. You, W.K. Cho, H.K. Shon, T.G. Lee, I.S. Choi, J.M. Karp, H. Lee, One step modification of superhydrophobic surfaces by a mussel inspired polymer coating, Angewandte Chemie International Edition, 49 (2010) 9401-9404. 
[27] A. Hozumi, O. Takai, Preparation of ultra water-repellent films by microwave plasma-enhanced CVD, Thin Solid Films, 303 (1997) 222-225.

[28] S. Mahadik, D.B. Mahadik, M.S. Kavale, V.G. Parale, P.B. Wagh, H. Barshilia, S. Gupta, N.D. Hegde, A.V. Rao, Thermally stable and transparent superhydrophobic solgel coatings by spray method, Journal of Sol-Gel Science and Technology, 63 (2012) 580-586.

[29] N.J. Shirtcliffe, G. McHale, M. I Newton, The superhydrophobicity of polymer surfaces: recent developments, Journal of Polymer Science Part B: Polymer Physics, 49 (2011) 1203-1217.

[30] S.H. Li, X.D. Sun, L.-j. Wang, W.-b. Gu, W.-m. Wang, Z.Y. Yang, Y. Wang, J.H. Zhu, Fabricating hydrophobic nanoparticles within mesoporous channel of silica for efficient TSNA removal, Microporous and Mesoporous Materials, 237 (2017) 237-245.

[31] G. Eris, D. Sanli, Z. Ulker, S.E. Bozbag, A. Jonás, A. Kiraz, C. Erkey, Threedimensional optofluidic waveguides in hydrophobic silica aerogels via supercritical fluid processing, J. Supercritical Fluids, 73 (2013) 28-33.

[32] P.B. Sarawade, J.-K. Kim, A. Hilonga, H.T. Kim, Preparation of hydrophobic mesoporous silica powder with a high specific surface area by surface modification of a wet-gel slurry and spray-drying, Powder Technology, 197 (2010) 288-294.

[33] A.P. Perissinotto, C.M. Awano, F.S. de Vicente, D.A. Donatti, A. Mesquita, L.F. da Silva, D.R. Vollet, Structure and diffuse-boundary in hydrophobic and sodium dodecyl sulfate-modified silica aerogels, Microporous and Mesoporous Materials, 223 (2016) 196-202. 
[34] J.L. Gurav, A.V. Rao, D.Y. Nadargi, H.H. Park, Ambient pressure dried TEOSbased silica aerogels: Good absorbents of organic liquids, J Mater Sci, 45 (2010) 503 510.

[35] A. Bisson, E. Rodier, A. Rigacci, D. Lecomte, P. Achard, Study of evaporative drying of treated silica gels, Journal of Non-Crystalline Solids, 350 (2004) 230-237.

[36] L.-J. Wang, S.-Y. Zhao, M. Yang, Structural characteristics and thermal conductivity of ambient pressure dried silica aerogels with one-step solvent exchange/surface modification, Materials Chemistry and Physics, 113 (2009) 485-490.

[37] A. Ślosarczyk, M. Barełkowski, S. Niemier, P. Jakubowska, Synthesis and characterisation of silica aerogel/carbon microfibers nanocomposites dried in supercritical and ambient pressure conditions, Journal of Sol-Gel Science and Technology, 76 (2015) 227-232.

[38] K. Kanamori, Liquid-phase synthesis and application of monolithic porous materials based on organic-inorganic hybrid methylsiloxanes, crosslinked polymers and carbons, Journal of Sol-Gel Science and Technology, 65 (2013) 12-22.

[39] W.J. Malfait, S. Zhao, R. Verel, S. Iswar, D. Rentsch, R. Fener, Y. Zhang, B. Milow, M.M. Koebel, Surface Chemistry of Hydrophobic Silica Aerogels, Chem. Mater., 27 (2015) 6737-6745.

[40] P. Patschinski, C. Zhang, H. Zipse, The Lewis Base-Catalyzed Silylation of Alcohols-A Mechanistic Analysis, The Journal of Organic Chemistry, 79 (2014) 83488357.

[41] P. Guo, S.-R. Zhai, Z.-Y. Xiao, F. Zhang, Q.-D. An, X.-W. Song, Preparation of superhydrophobic materials for oil/water separation and oil absorption using PMHS- 
TEOS-derived xerogel and polystyrene, Journal of Sol-Gel Science and Technology, 72 (2014) 385-393.

[42] N. Job, R. Pirard, J.P. Pirard, C. Alié, Non Intrusive Mercury Porosimetry: Pyrolysis of Resorcinol Formaldehyde Xerogels, Particle \& Particle Systems Characterization, 23 (2006) 72-81.

[43] K.P.C. Vollhardt, N.E. Schore, Organic Chemistry, W. H. Freeman 2010. 


\section{List of Table captions:}

Table 1. Porous properties of the two RF xerogels studied

\section{List of Figure captions:}

Figure 1. Grafting reaction mechanism between RF xerogel surface groups and hexamethyldisilazane (HMDZ).

Figure 2. Si (2p) high-resolution XPS spectra of the RF xerogel OX-60 and its counterpart treated with HMDZ (Si-OX-60).

Figure 3. Evolution of $\mathrm{m} / \mathrm{z} 75$, corresponding to trimethylsyloxyl compounds $\left(\mathrm{C}_{2} \mathrm{H}_{7} \mathrm{SiO}^{+}\right)$, during TGA-MS analysis for the RF xerogel OX-60 and its counterpart treated with HMDZ (Si-OX-60).

Figure 4. Evolution of the contact angle, as measured by an optical tensiometer, with grafting reaction times for the silanes tested. Si-OX samples are RF xerogels grafted with HMDZ (see text).

Figure 5. Pore size distribution of the RF xerogels before and after treatment with HMDZ.

Figure 6. Loss of hydrophobicity with time of exposure under ambient humid conditions $\left(20^{\circ} \mathrm{C}\right.$ and $80 \%$ humidity).

Figure 7. Proof of hydrophobicity of RF xerogels grafted with silanes (a). Materials become hydrophilic again after overnight shaking of the water suspension (b). 


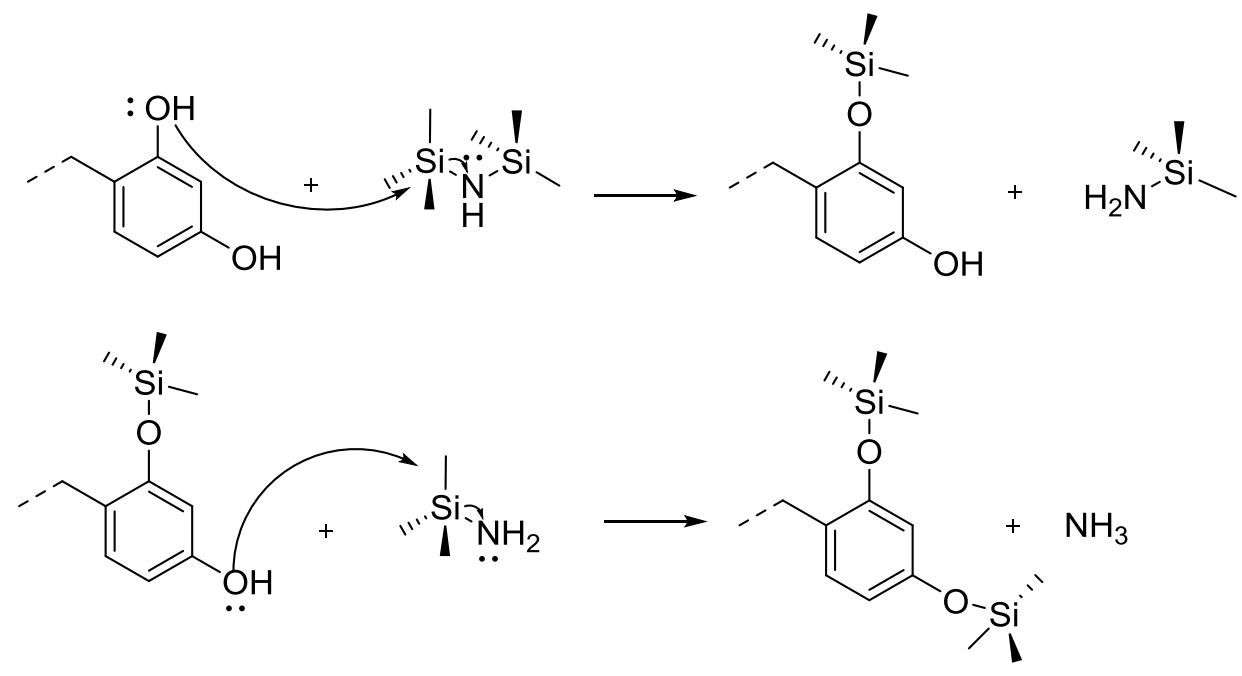

Figure 1. Grafting reaction mechanism between RF xerogel surface groups and hexamethyldisilazane (HMDZ). 


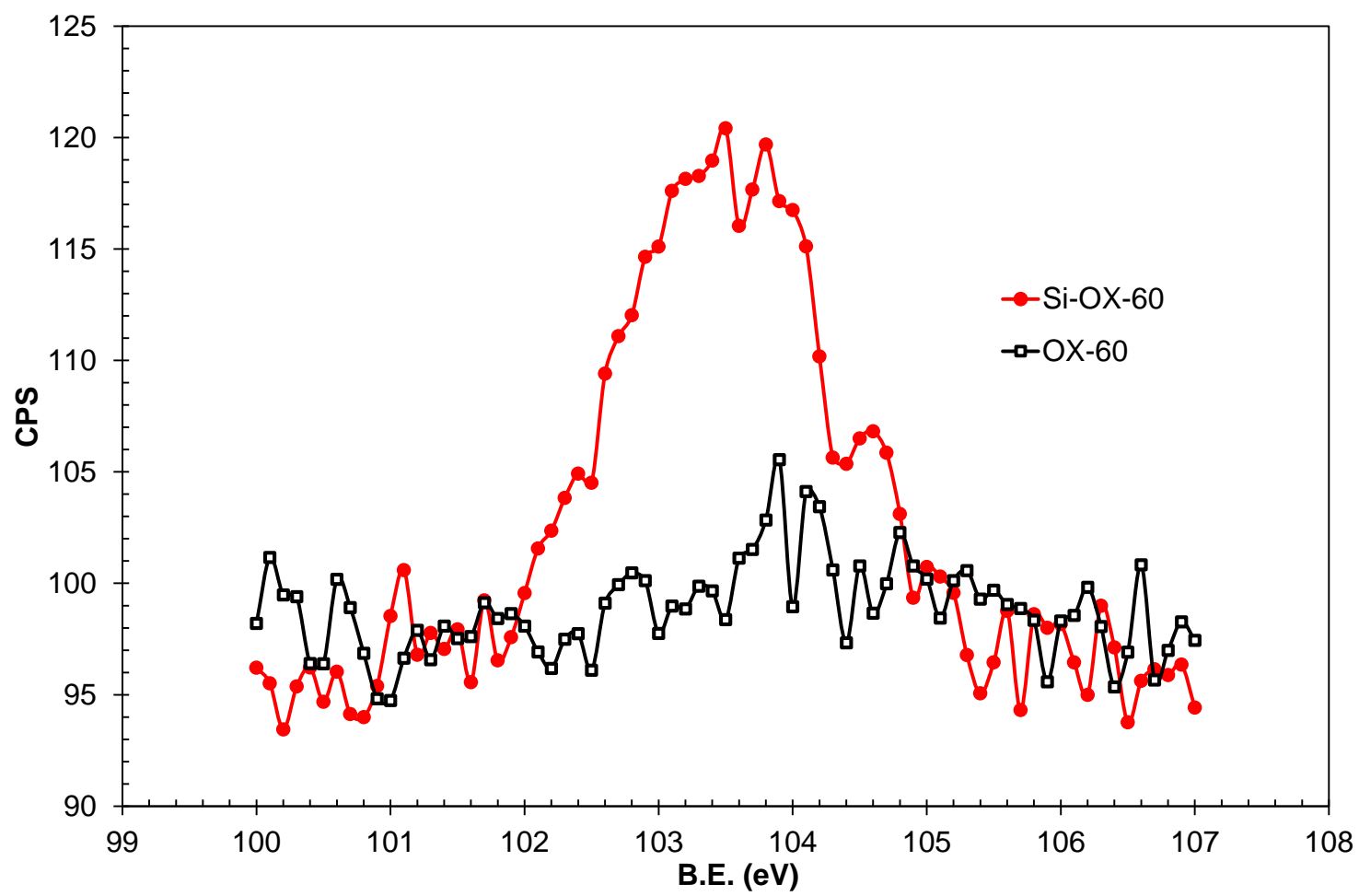

Figure 2. Si (2p) high resolution XPS spectra of the RF xerogel OX-60 and its counterpart treated with HMDZ (Si-OX-60). 


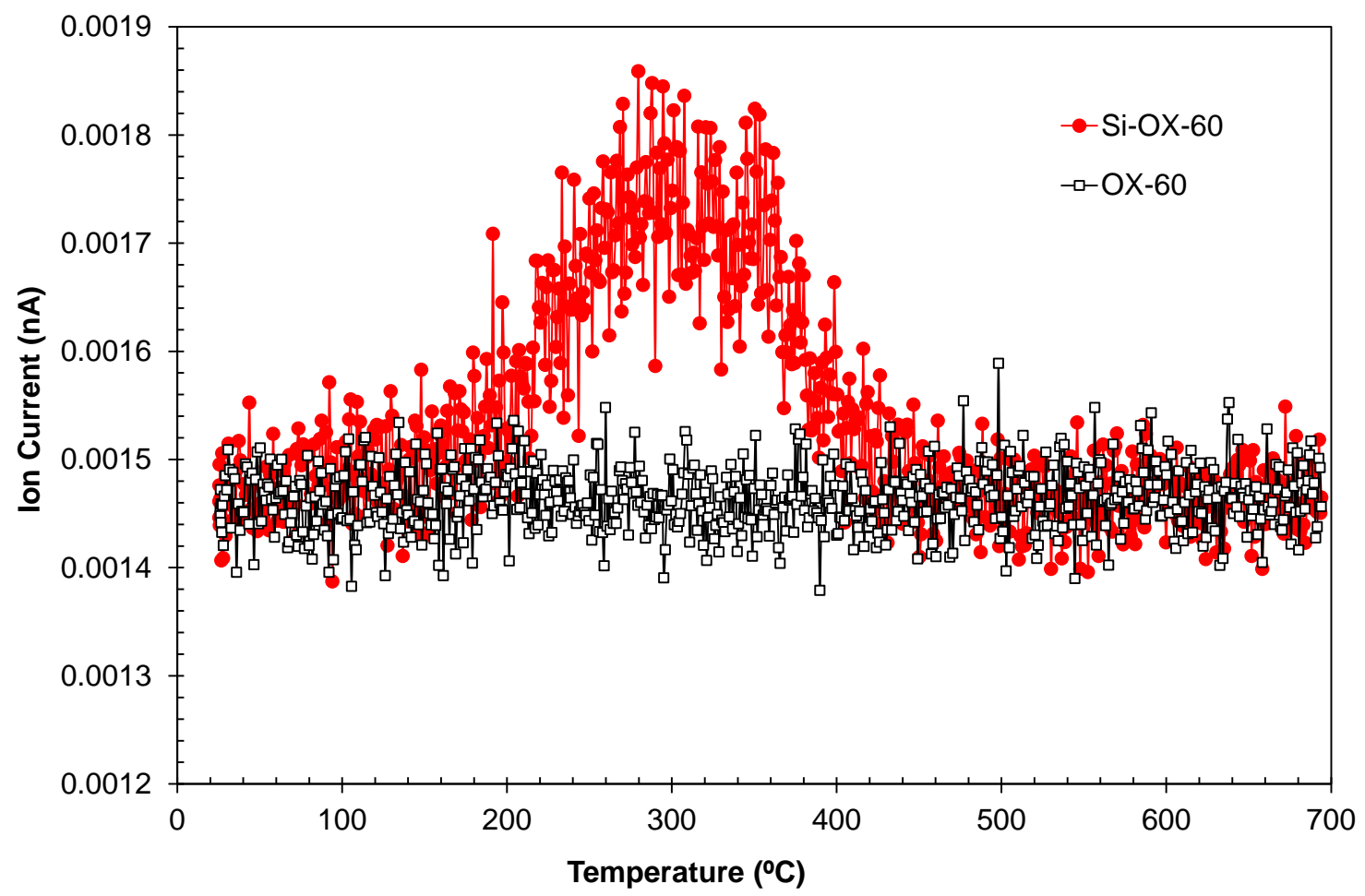

Figure 3. Evolution of $\mathrm{m} / \mathrm{z} 75$, corresponding to trimethylsyloxyl derivatives, during TGA-MS analysis for the RF xerogel OX-60 and its counterpart treated with HMDZ (Si-OX-60). 


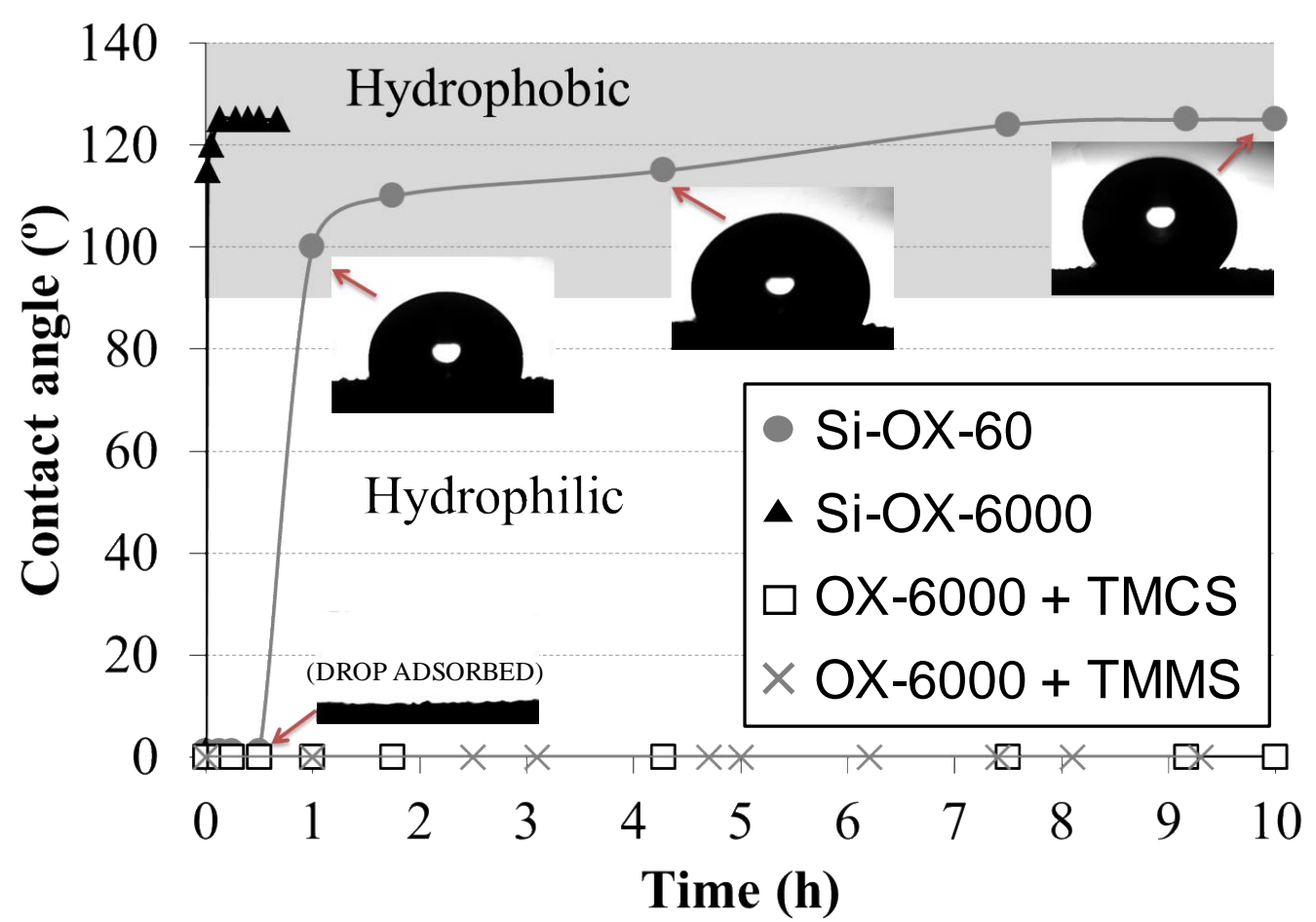

Figure 4. Evolution of the contact angle, as measured by an optical tensiometer, with grafting reaction times for the silanes tested. Si-OXs samples are RF xerogels grafted with HMDZ (see text). 


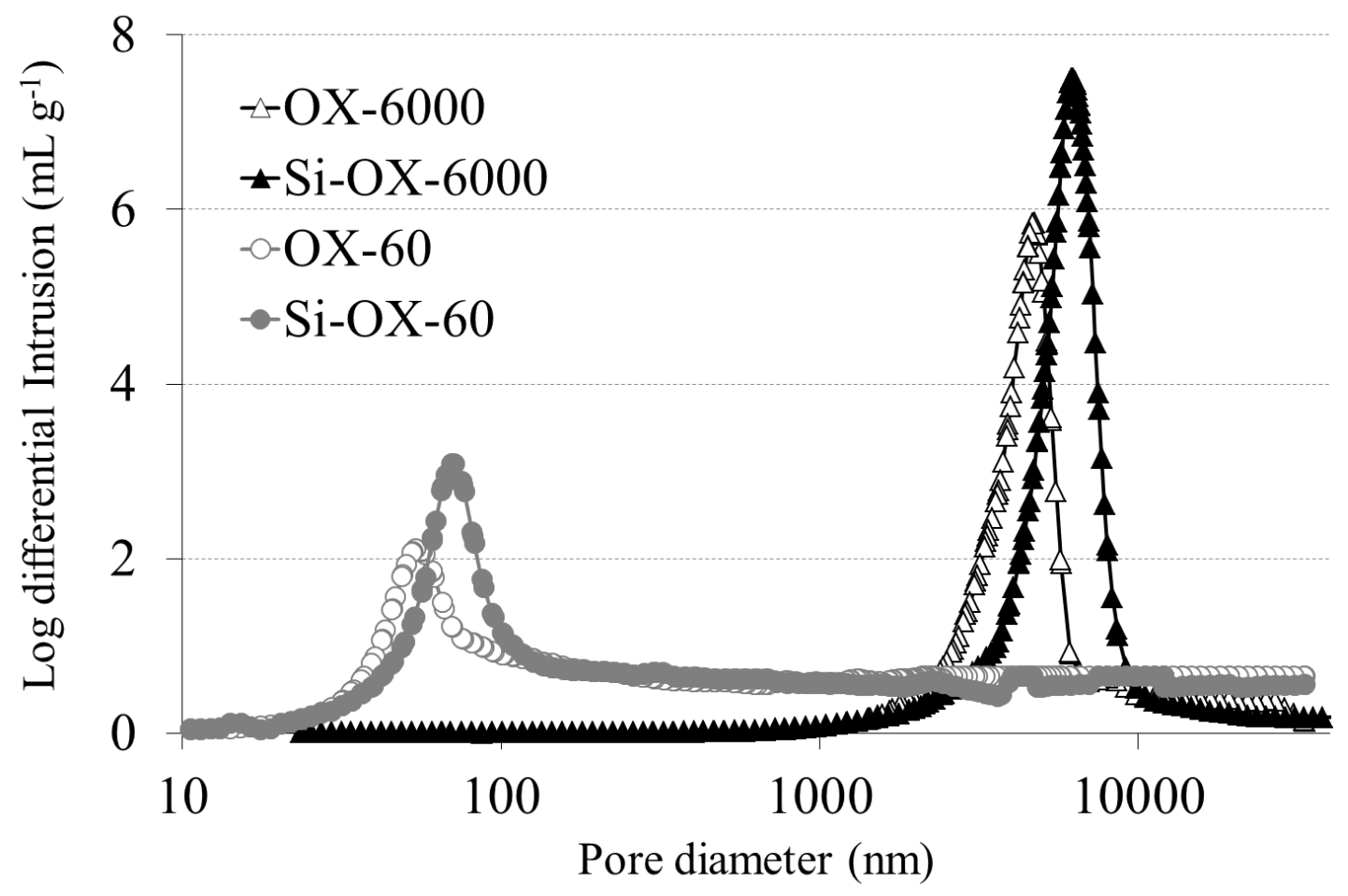

Figure 5. Pore size distribution of the RF xerogels before and after treatment with HMDZ. 


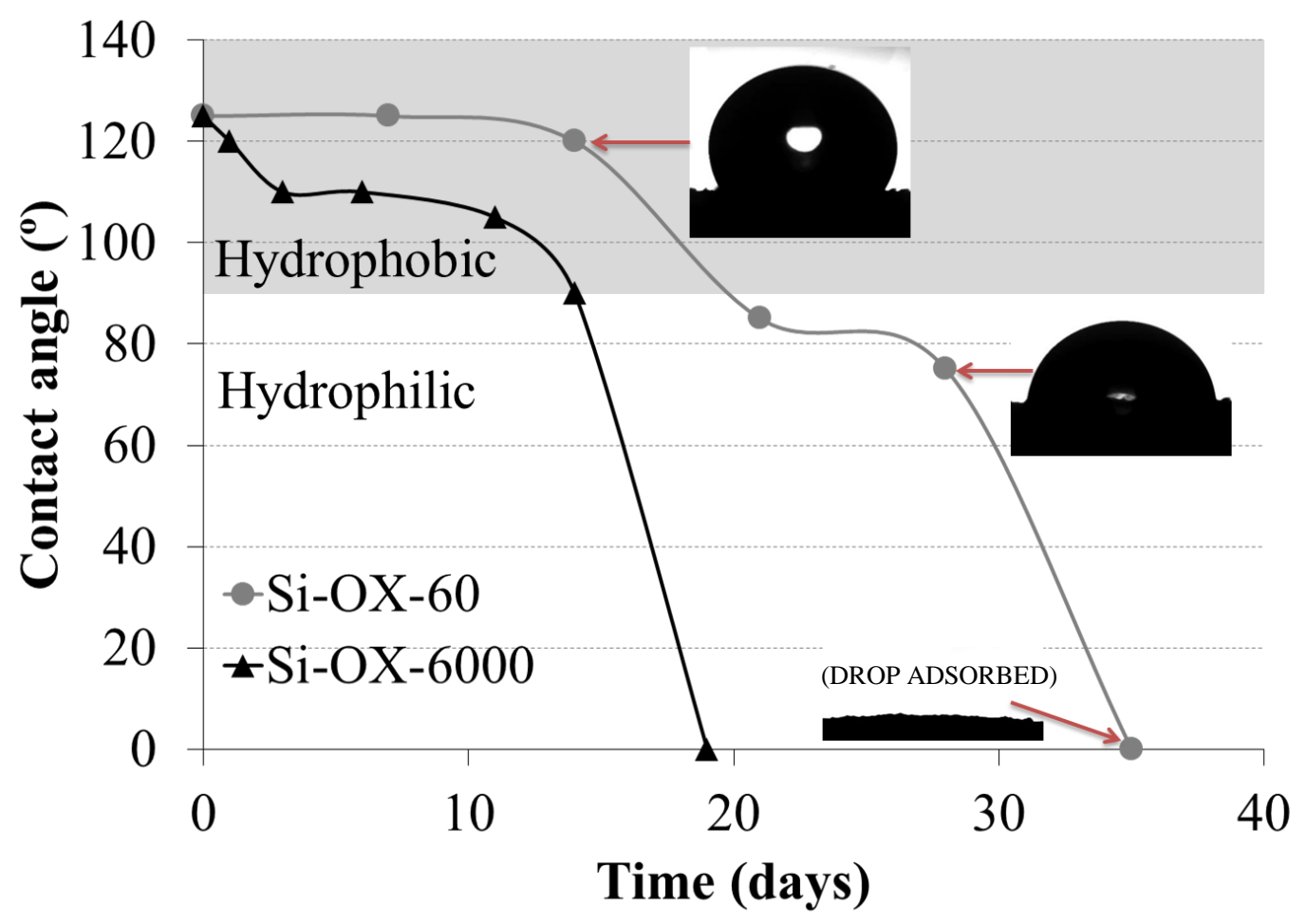

Figure 6. Loss of hydrophobicity with time of exposure to ambient humid conditions ( $20^{\circ} \mathrm{C}$ and $80 \%$ humidity). 


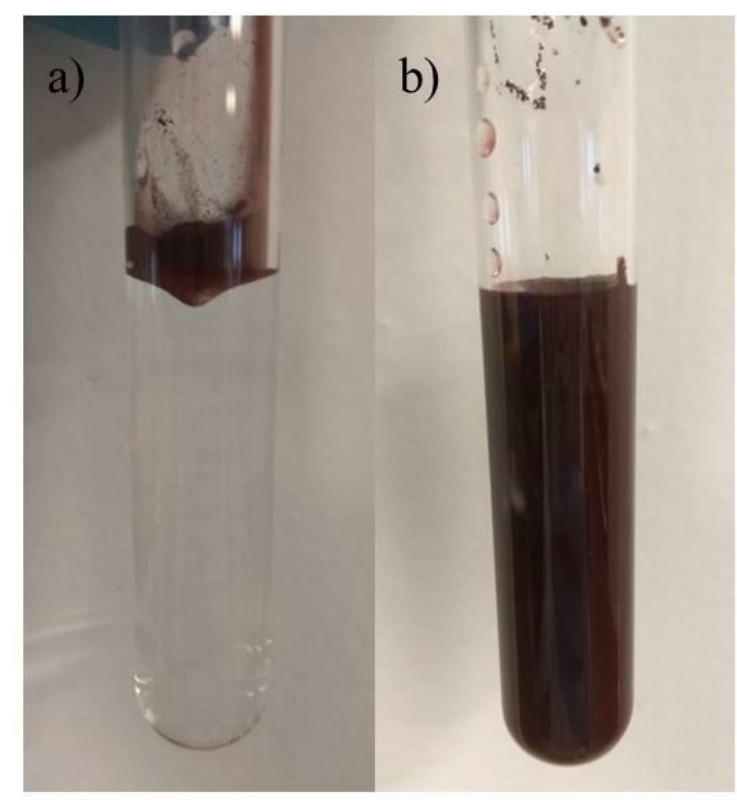

Figure 7. Proof of hydrophobicity of RF xerogels grafted with silanes (a). The materials become hydrophilic again after overnight shaking of the water suspension (b). 